\title{
Throughput Estimation with Noise Uncertainty for Cyclostationary Feature Detector in Cognitive Radio Network
}

\author{
Mohsen M. Tantawy \\ National Telecommunication Institute, Cairo, Egypt \\ Email: ntimohsen@gmail.com
}

Received 11 May 2015; accepted 2 June 2015; published 5 June 2015

Copyright (C) 2015 by author and Scientific Research Publishing Inc. This work is licensed under the Creative Commons Attribution International License (CC BY). http://creativecommons.org/licenses/by/4.0/ (c) (7) Open Access

\begin{abstract}
Cognitive Radio Networks (CRNs) are recognized as the enabling technology for improving the future bandwidth utilization. In CRNs secondary users are allowed to utilize the frequency bands of primary users when these bands are not currently being used. The secondary users are required to sense the radio frequency environment. The lower the probability of false alarm, the more chances the channel can be reused and the higher the achievable throughput for the secondary network. The main contribution of this paper is to formulate the sensing-throughput-noise uncertainty tradeoff for cyclostationary feature detection. Computer simulations have shown that for a $1 \mathrm{MHz}$ channel, when the sensing duration is $2 \%$ of total time, the spectrum will get $99 \%$ probability of detection regardless of $50 \%$ noise uncertainty.
\end{abstract}

\section{Keywords}

Cognitive Radio Network, Cyclostationary Feature Detector, Throughput, Noise Uncertainty

\section{Introduction}

Cognitive radio networks are one of the enabling technologies for future communication and networking [1]. Cognitive radio depends on Dynamic Spectrum Access (DSA) which allows secondary users (SUs) to occupy the unused spectrum instead of keeping it idle or unused by the primary user (PU) [2]. The first function of the cognitive radio networks which is called the spectrum sensing senses the free spectrum bands and the presence or absence of the primary user [3] [4]. After sensing the spectrum management will take place by selecting the best band, spectrum sharing and allocation by coordinating fair spectrum access to this channel with other users, and finally spectrum mobility by vacating the channel when a licensed user is detected. 
Spectrum sensing can be divided into two main categories cooperative detection technique and noncooperative. In the cooperative detection secondary users collect the information regarding channel occupancy and transmit this information into spectrum map that updated periodically. In non-cooperative detection, individual radios act locally and autonomously to carry out their own spectrum occupancy measurements and analysis. It can be divided into two classes blind sensing which does not need any information about the primary user signal such as energy detector, and signal specific sensing which need information about the primary user signal such as matched filter and feature detector [3].

According to IEEE 802.22 wireless regional area networks (WRAN), each medium access control (MAC) frame consists of one sensing slot and one data transmission slot, and thus periodic spectrum sensing should be carried out such that the cognitive radio users can decide whether the next frame can continue to transmit on the spectrum band [5] [6]. Associated with spectrum sensing are two parameters: probability of detection and probability of false alarm. The higher the detection probability, the better the primary users can be protected. However, from the secondary users' perspective, the lower the false alarm probability, the more chances the channel can be reused when it is available, thus the higher the achievable throughput for the secondary users. Thus there could exist a fundamental tradeoff between sensing capability and achievable throughput for CR different detectors [7].

This paper is organized as follows: Section 2 investigates the related work in throughput in cyclostationary feature detectors in CRN; Section 3 discusses the system model and its assumptions; Section 4 presents the numerical results; and Section 5 gives the conclusion and the future work.

\section{Related Work}

CRN has to enforce quiet periods to effectively sense the spectrum availability to protect the PUs. Spectrum sensing and sensing time length can vary depending on the algorithm according to [8]. In cooperative sensing, the length of sensing time at individual SUs is proportional to the sensing accuracy increasing sensing time decreases the transmission time. The trade-off is called the sensing efficiency problem and is discussed in [9] and [10]. In [11] the CRN system throughput is maximized by cooperative sensing. Optimal spectrum sensing time considering spectrum handoff due to false alarm discussed in [12]. As in [13], sensing decision problem for maximizing the system throughput is discussed. Also in [13] a suggested algorithm to maximizing the throughput by cooperative sensing is suggested. In [14], maximizing secondary network throughput while keeping under control the average interference is formulated. Authors in [15] study the effect of noise uncertainty on choosing the minimum number of samples in energy and matched filter detectors. The authors in [16] propose a fast spectrum detecting algorithm based on cyclic autocorrelation of communication signals and select the users with good detection performance to cooperative sense to improve sensing sensitivity without considering noise uncertainty. In [17] a comparison of sensing algorithms which revealed wide variability in their computational complexity for the targeted detection performance without considering network throughput is presented. Authors in [18] propose novel spectrum sensing algorithm, and examines the sensing throughput tradeoff for energy detector in CRN under noise variance uncertainty.

\section{System Model}

The problem of signal detection in additive Gaussian noise can be formulated as a binary hypothesis testing problem with the following hypotheses:

$$
\left\{\begin{array}{l}
H_{0}: X(n)=V(n), n=1,2, \cdots, N ; \\
H_{1}: X(n)=S(n)+V(n), n=1,2, \cdots, N
\end{array}\right.
$$

where $X(n), S(n)$ and $V(n)$ are the received signals at CR nodes, transmitted signals at primary nodes and white noise samples, respectively; $H_{1}$ and $H_{0}$ stand for the decision that the licensed user is present or not, respectively. Noise samples $V(n)$ are from additive white Gaussian noise process with power spectral density $\sigma^{2}$ [19].

Feature detector in CR network operates in the mid-way between Energy and Matched filter detectors. The cyclostationary feature detector relies on the fact that most signals exhibit periodic features, present in pilots, cyclic prefixes, modulations, carriers, and other repetitive characteristics. Because the noise is not periodic, the signal can be successfully detected [16]-[18]. 
The feature detector correlates received signal $X(n)$ with frequency shifted version of itself resulting in spectral correlation function (SCF) [19].

$$
S_{\alpha}^{X}(f)=\frac{1}{N K} \sum_{n=1}^{N} X_{K}\left(n, f+\frac{\alpha}{2}\right) X_{K}^{*}\left(n, f-\frac{\alpha}{2}\right)
$$

Here $X_{K}(n, f)$ is the $K$ point FFT around sample $n$ and $\alpha$ is the amount of frequency shift. The decision statistic is modeled in this SCF and it is compared against given threshold $\gamma$.

$$
T(X)=\sum_{n=1}^{N} S_{\alpha}^{X}(f)>\gamma
$$

The probability of false alarm and the probability of detection can be given as [19]

$$
\begin{gathered}
P_{f}=\mathrm{e}^{\frac{-N \gamma^{2}}{2 \sigma^{4}}} \\
P_{d}=Q_{m}\left(\frac{\psi}{\sigma_{T}}, \frac{\gamma}{\sigma_{T}}\right)
\end{gathered}
$$

where, $\psi=\frac{K P_{S}}{2}, \sigma_{T}=\sqrt{\frac{K P_{S} \sigma^{2}}{N}+\frac{\sigma^{4}}{N}}$, FT points [19]. And $Q_{m}($.$) is the first order Marcum Q$ functionaccording to [20] and given in Equation (6).

$$
Q_{m}(a, b)=\int_{b}^{\infty} x \exp \left(-\frac{x^{2}+a^{2}}{2}\right) I_{0}(a x) \mathrm{d} x, a \geq 0, b \geq 0
$$

where $I_{0}($.$) is the zeroth-order modified Bessel function given in Equation (7)$

$$
I_{0}(y)=\frac{1}{\pi} \int_{0}^{\infty} \exp (y \cos \theta) \mathrm{d} \theta
$$

Solving for $\gamma$ to get detection probability

$$
P_{d}=Q_{m}\left(\frac{S N R K \sqrt{N}}{2 \sqrt{S N R K+1}}, \sqrt{\frac{-2 \ln P_{f}}{S N R K+1}}\right) .
$$

Denote $C_{0}$ as the throughput of the secondary network in the absence of PU, $C_{1}$ is the throughput in the presence of PU, so the throughput $C$ of the secondary network according to [21] will given by

$$
C=\left\{\begin{array}{l}
C_{0}=\log _{2}\left(1+S N R_{S}\right) \\
C_{1}=\log _{2}\left(1+\frac{P_{s}}{P_{p}+N_{0}}\right)=\log _{2}\left(1+\frac{S N R_{s}}{S N R_{p}+1}\right) .
\end{array}\right.
$$

The normalized rate for unit bandwidth for the secondary network with sensing slot time $\tau$ and a frame duration time $T$ under hypothesis $H_{0}$ and $H_{1}$ are given by

$$
R=\left\{\begin{array}{l}
R_{0}=\frac{T-\tau}{T} C_{0}\left(1-P_{f}(\gamma, \tau)\right) p\left(H_{0}\right) \\
R_{1}=\frac{T-\tau}{T} C_{1}\left(1-P_{d}(\gamma, \tau)\right) p\left(H_{1}\right) .
\end{array}\right.
$$

And the average throughput for the secondary network will given by

$$
\begin{aligned}
& R(\tau)=R_{0}(\gamma, \tau)+R_{1}(\gamma, \tau) \\
& R(\tau)=\frac{T-\tau}{T} C_{0}\left(1-P_{f}(\gamma, \tau)\right) p\left(H_{0}\right)+\frac{T-\tau}{T} C_{1}\left(1-P_{d}(\gamma, \tau)\right) p\left(H_{1}\right) .
\end{aligned}
$$


For cyclostationary feature detector $P_{f}$ and $P_{d}$ are given from Equations (4) and (5) then the average throughput for the secondary network will be

$$
\begin{aligned}
R(\tau)= & \frac{T-\tau}{T} \log _{2}\left(1+S N R_{S}\right)\left(1-P_{f}\right) p\left(H_{0}\right)+\frac{T-\tau}{T} \log _{2}\left(1+\frac{S N R_{S}}{S N R_{P}+1}\right) \\
& \times\left(1-Q_{m}\left(\frac{S N R_{P} K \sqrt{N}}{2 \sqrt{S N R_{p} K+1}}, \sqrt{\frac{-2 \ln P_{f}}{S N R_{P} K+1}}\right)\right) p\left(H_{1}\right)
\end{aligned}
$$

Due to noise uncertainty, the estimated noise power may be different from the actual noise power. Let the estimated noise power changed in the interval $\sigma_{v}^{2} \in\left[\sigma^{2} / \beta, \beta \sigma^{2}\right]$ where $\beta>1$ is the noise uncertainty factor. For feature detector and in a noise variation environment, minimizing the Marcum $Q$ function in $P_{d}$ and maximizing $P_{f}$, with threshold $\gamma$, hence

$$
P_{f}=\mathrm{e}^{\frac{-N \gamma}{2 \sigma^{4} \beta^{2}}}
$$

The threshold will given by

$$
\gamma=\sqrt{\frac{-2\left(\sigma^{4} \beta^{2}\right) \ln P_{f}}{N} .}
$$

According to [22] and Equation (5) when $\frac{\psi}{\sigma_{T}}$ decreased and $\frac{\gamma}{\sigma_{T}}$ increased the value of Marcum $Q$ function decreased

$$
P_{d}=Q_{m}\left(\frac{\frac{S N R}{\beta} K \sqrt{N}}{2 \sqrt{\frac{S N R}{\beta} K+1}}, \sqrt{\frac{-2 \ln P_{f}}{\frac{S N R}{\beta} K+1}}\right) .
$$

Modifying (12) to show the effect of noise variation on throughput in cyclostationary feature detector will give

$$
\begin{aligned}
R(\tau)= & \frac{T-\tau}{T} \log _{2}\left(1+S N R_{S}\right)\left(1-P_{f}\right) p\left(H_{0}\right)+\frac{T-\tau}{T} \log _{2}\left(1+\frac{S N R_{S}}{S N R_{P}+1}\right) \\
& \times\left(1-Q_{m}\left(\frac{\frac{S N R_{p}}{\beta} K \sqrt{N}}{2 \sqrt{\frac{S N R_{p}}{\beta} K+1}}, \sqrt{\frac{-2 \ln P_{f}}{\frac{S N R_{p}}{\beta} K+1}}\right)\right) p\left(H_{1}\right)
\end{aligned}
$$

where $N=f_{s} \tau$ and $P_{f}$ is given by Equation (13).

\section{Numerical Results}

In this section, numerical results are presented to evaluate the sensing-throughput trade-off with noise uncertainty for feature detector scheme. Table 1 summarizes the numerical values used in the paper according to [7] and [8].

Probability of detection versus probability of false alarm with $S N R$ variation for feature detector is shown in Figure 1. As it could expected, the performance of feature detector decreases with $S N R$. The receiver operating characteristic curve (ROC) with noise uncertainty is shown in Figure 2. Curves displayed in Figure 2 are calculated numerically. Value of $S N R$ is $-20 \mathrm{~dB}$ with 1 ms sensing time and corresponding values of $P_{d}$ are calculated with Equation (13). The noise uncertainty factor of receiving device is normally up to 1.585 [23]. 
Table 1. System parameters.

\begin{tabular}{cc}
\hline System parameters & Value \\
\hline$P\left(H_{0}\right)$ & 0.8 \\
$P\left(H_{1}\right)$ & 0.2 \\
Sampling frequency $f_{s}$ & $10^{6} \mathrm{~Hz}$ \\
$K$ is the number of FFT points & 8 \\
Frame duration $(T)$ & $100 \mathrm{~ms}$ \\
Sensing duration & $1 \mathrm{~ms}: 25 \mathrm{~ms}$ \\
SNR & {$[0,-30] \mathrm{dB}$} \\
Probability of false alarm $\left(P_{f}\right)$ & {$[0,0.5]$} \\
Noise uncertainty factor $(\beta)$ & Up to 1.5 \\
\hline
\end{tabular}

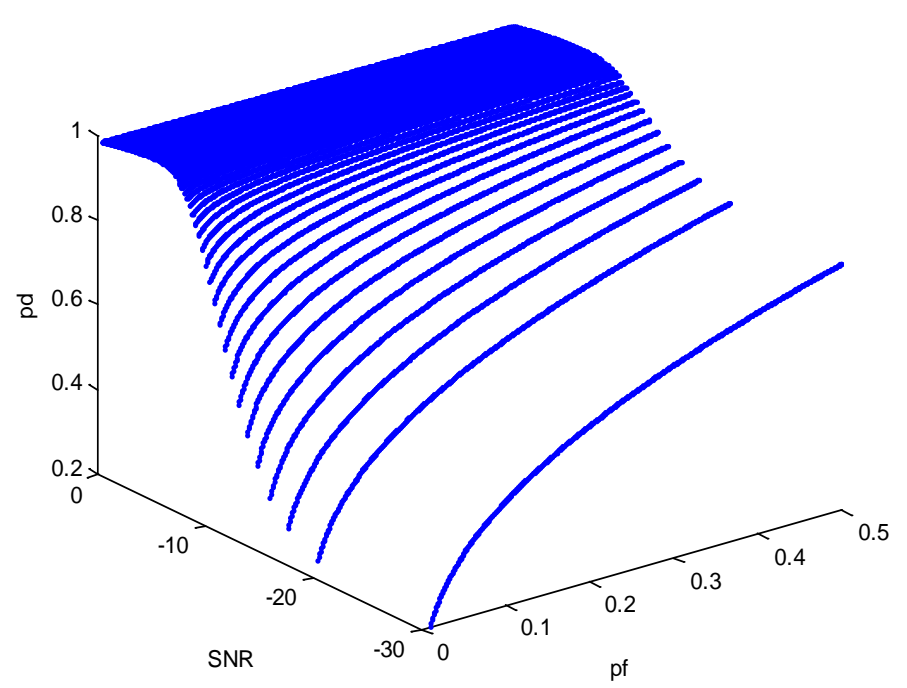

Figure 1. Effect of SNR on the feature detector receiver operating characteristics.

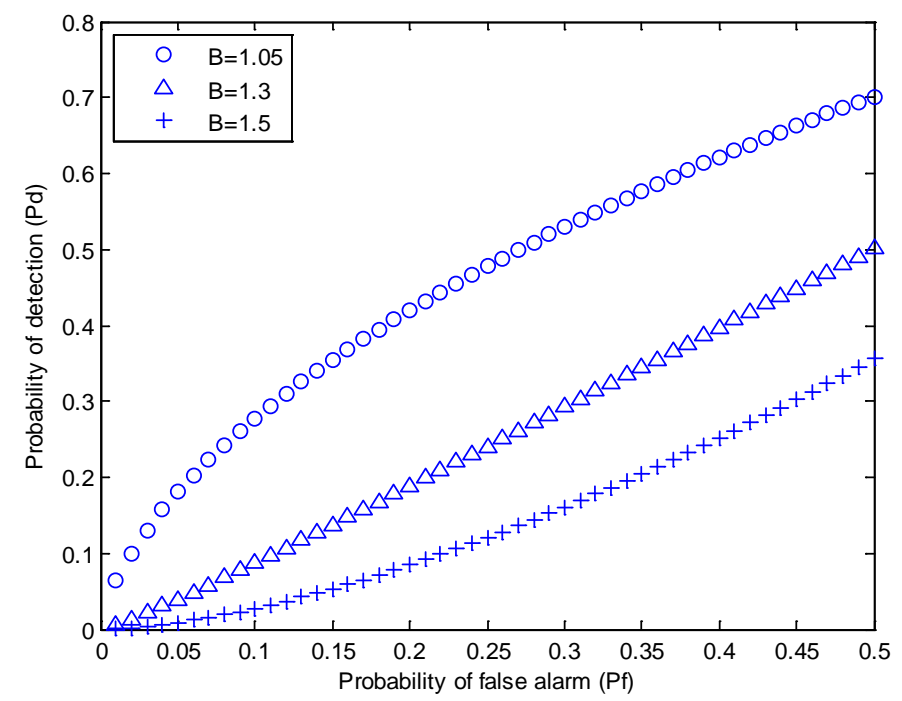

Figure 2. Receiver operating characteristics of feature detector with noise uncertainty. 
The effect of different noise uncertainty factor values on probability of detection with variable SNR values with 0.01 false alarm probability is shown in Figure 3. The figure shows that $S N R$ value between -18 to $-20 \mathrm{~dB}$ has dramatically effect on detection probability.

Figure 4 shows the effect of sensing time on normalized secondary network throughput with $-20 \mathrm{~dB} S N R$ value, and 1.5 noise uncertainty factor. Figure 5 shows that $20 \mathrm{~ms}$ sensing time or spending $2 \%$ of total time to sense the spectrum will get $99 \%$ probability of detection regardless of $50 \%$ noise uncertainty change.

Figure 6 shows the effect of noise uncertainty on the secondary network throughput with $-20 \mathrm{~dB} S N R$, and 0.01 probability of false alarm. The figure shows that increasing the uncertainty factor from 1.05 to 1.5 leads to changing the overall throughput by $8 \%$.

\section{Conclusion and Future Work}

The effect of noise uncertainty on the secondary network throughput with low SNR values is analyzed. Moreover,

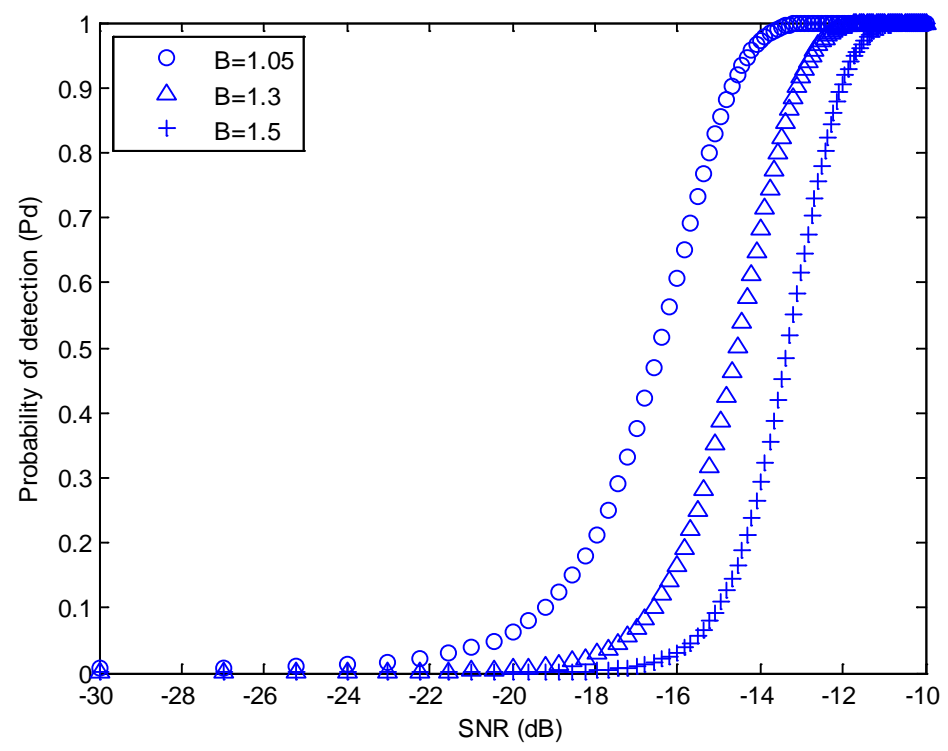

Figure 3. $S N R$ versus $P d$ with variable noise uncertainty factor.

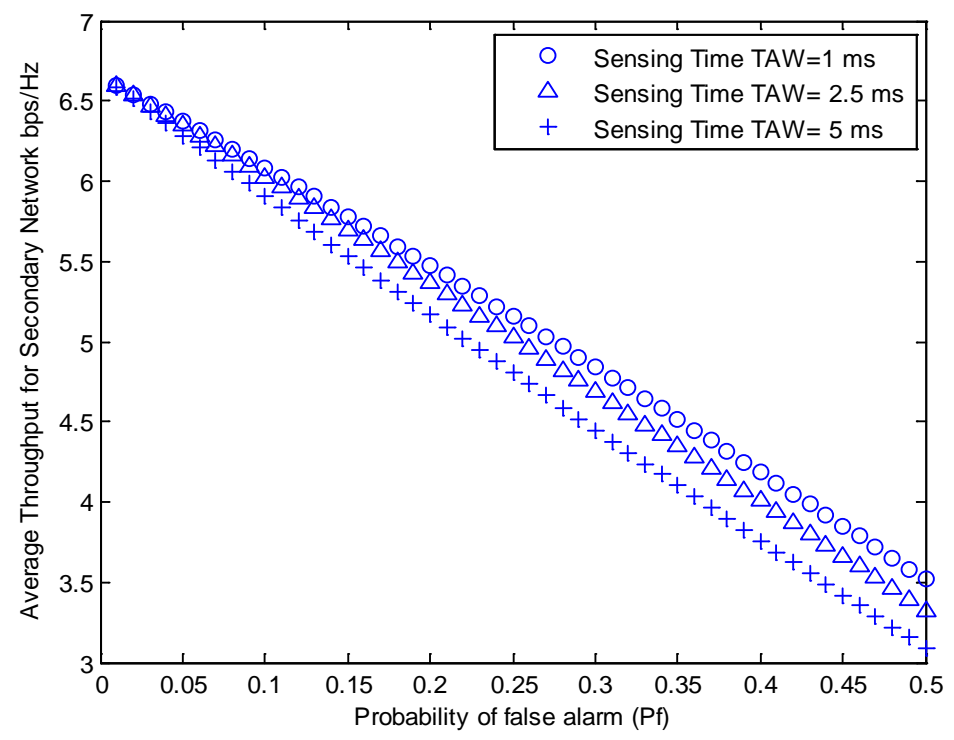

Figure 4. Effect of sensing time on normalized secondary network throughput. 


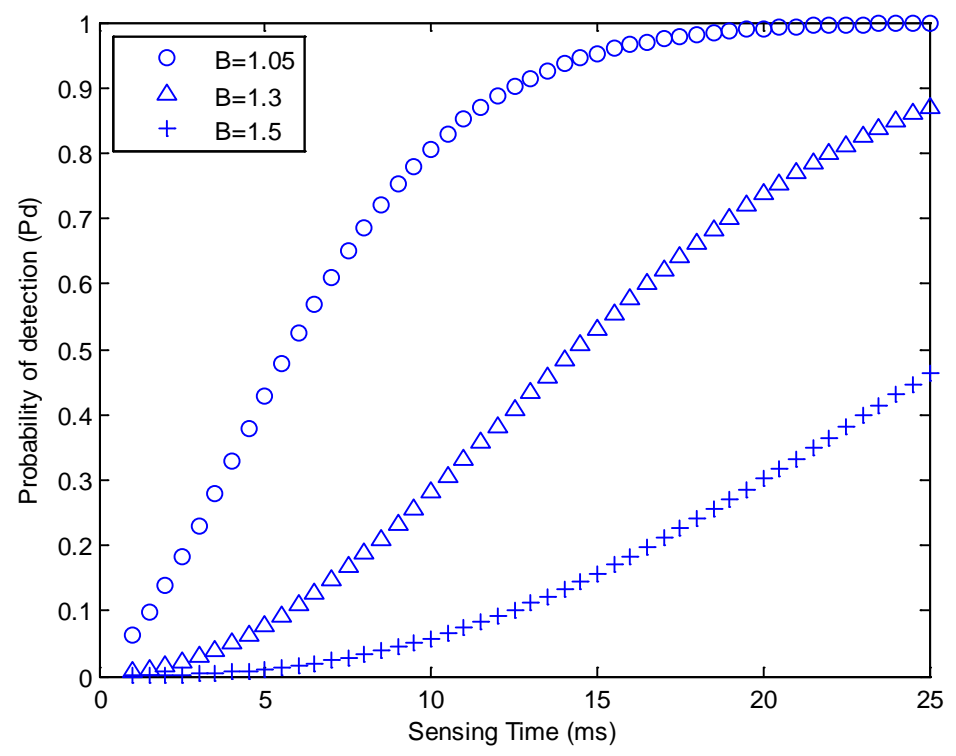

Figure 5. Probability of detection $(P d)$ versus sensing time for with noise uncertainty.

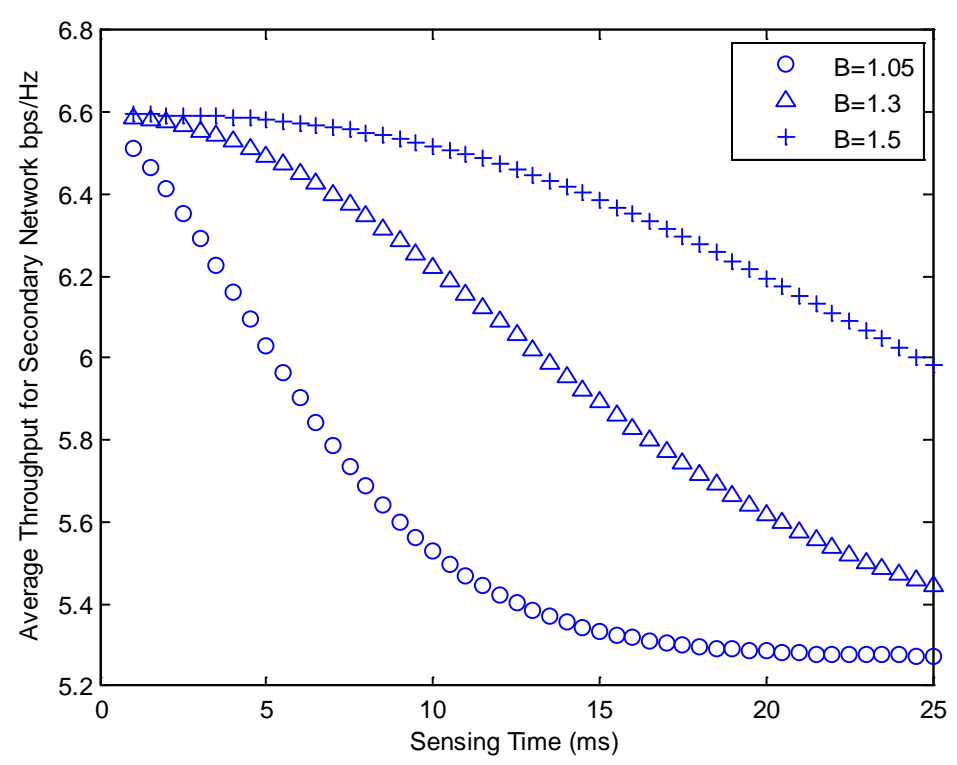

Figure 6. Normalized secondary network throughput versus sensing time with noise uncertainty.

selecting the sensing time with the proper value of SNR leads to better overall normalized throughput. The contribution of this paper is that increasing the noise uncertainty by $50 \%$ decreases throughput by $8 \%$. On the other hand, noise uncertainty of $50 \%$ can be overcome if the sensing time reaches $2 \%$ of the total time. This work can be extended to include eigenvalue based detector.

\section{References}

[1] Akyildiz, I.F., Lee, W.-Y., Vuran, M.C. and Mohanty, S. (2006) Next Generation/Dynamic Spectrum Access/Cognitive Radio Wireless Networks: A Survey. Computer Network, 50, 2127-2159. http://dx.doi.org/10.1016/j.comnet.2006.05.001

[2] Zhang, Y., Zheng, J. and Chen, H.-H. (2010) Cognitive Radio Networks Architectures, Protocols, and Standards. http://dx.doi.org/10.1201/EBK1420077759 
[3] Imam, M.S., Ingle, S. and Ara, S. (2013) A Review Paper Based on Spectrum Sensing Techniques in Cognitive Radio Networks. IISTE, 3.

[4] Thakre, S. and Dixit, S. (2014) Security Threats and Detection Technique in Cognitive Radio Network with Sensing Strategies. IJRET, 3.

[5] (2005) IEEE 802.22 Wireless RAN, Functional Requirements for the 802.22 WRAN Standards, IEEE802.22-05/ $0007 \mathrm{r} 46$.

[6] Pei, E., Li, J.B. and Cheng, F. (2013) Sensing-Throughput Tradeoff for Cognitive Radio Networks with Additional Primary Transmission Protection. Journal of Computational Information Systems, 9, 3767-3773.

[7] Liang, Y.-C., Zeng, Y.H., Peh, E.C.Y. and Hoang, A.T. (2008) Sensing-Throughput Tradeoff for Cognitive Radio Networks. IEEE Transactions on Wireless Communications, 7, 1326-1337. http://dx.doi.org/10.1109/TWC.2008.060869

[8] Yucek, T. and Arslan, H. (2009) A Survey of Spectrum Sensing Algorithms for Cognitive Radio Applications. IEEE Communications Surveys \& Tutorials, 11, 116-130. http://dx.doi.org/10.1109/SURV.2009.090109

[9] Lee, W. (2008) Optimal Spectrum Sensing Framework for Cognitive Radionetworks. IEEE Transactions on Wireless Communications, 7, 3845-3857. http://dx.doi.org/10.1109/T-WC.2008.070391

[10] Min, A. and Shin, K. (2009) An Optimal Sensing Framework Based on Spatialrss-Profile in Cognitive Radio Networks. Proceedings of SECON, Piscataway, 207-215.

[11] Faisal Amjad, M., Aslam, B. and Zou, C.C. (2012) Transparent Cross-Layer Solutions for Throughput Boost in Cognitive Radio Networks. Consumer Communications and Networking Conference, Las Vegas, 14-17 January 2012.

[12] Lee, D.-J. and Jang, M.-S. (2009) Optimal Spectrum Sensing Time Considering Spectrum Handoff Due to False Alarm in Cognitive Radio Networks. IEEE Communications Letters, 13, 899-901. http://dx.doi.org/10.1109/LCOMM.2009.12.091448

[13] Li, S., Zheng, Z.Z., Ekici, E. and Shroff, N. (2014) Maximizing System Throughput by Cooperative Sensing in Cognitive Radio Networks. IEEE/ACM Transactions on Networking, 22, 1245-1256. http://dx.doi.org/10.1109/TNET.2013.2272722

[14] Shokri-Ghadikolaei, H., Forough, Y. and Carlo, F. (2014) Analysis and Optimization of Centralized Sequential Channel Sensing in Cognitive Radio Networks. 20th European Wireless Conference, Barcelona, 14-16 May 2014, 1-6.

[15] Tantawy, M. (2014) Responsive Communication Jamming Detector with Noise Power Fluctuation Using Cognitive Radio. International Journal of Innovation Research in Computer and Communication Engineering, 2, 5967-5973.

[16] Ou, Y. and Wang, Y.-M. (2012) Performance of Spectrum Sensing and Optimization Based on User Selection in Cognitive Radio. International Journal of Communications, 6.

[17] Umar, R. and Shekha, A.U.H. (2012) A Comparative Study of Spectrum Awareness Techniques for Cognitiveradio Oriented Wireless Networks. Elsevier Physical Communication Journal, 1-23.

[18] Bogale, T.E., Vandendorpe, L. and Le, L.B. (2014) Sensing Throughput Tradeoff for Cognitive Radio Networks with Noise Variance Uncertainty. CROWNCOM 2014-9th International Conference on Cognitive Radio Oriented Wireless Networks, Oulu, 2-4 June 2014.

[19] Cabric, D., Tkachenko, A. and Brodersen, R.W. (2006) Experimental Study of Spectrum Sensing Based on Energy Detection and Netwotk Cooperation. International Workshop on Technology and Policy for Accessing Spectrum, Boston. http://dx.doi.org/10.1145/1234388.1234400

[20] Corazza, G. and Ferrari, G. (2002) New Bounds for the Marcum Q-Function. IEEE Transactions on Information Theory, 48, 3003-3008. http://dx.doi.org/10.1109/TIT.2002.804113

[21] Cognitive Radio Networks (CRNs). University of Waterloo, Department of Electrical and Computer Engineering, Waterloo.

[22] Bocus, M.Z., Dettmann, C.P. and Coon, J.P. (2013) An Approximation of the First Order Marcum Q-Function with Application to Network Connectivity Analysis. IEEE Communication Letters, 17, 499-502. http://dx.doi.org/10.1109/LCOMM.2013.011513.122462

[23] Zeng, Y.H. and Liang, Y.C. (2009) Eigenvalue Based Spectrum Sensing Algorithms for Cognitive Radio. IEEE Transactions on Communications, 57, 1784-1793. http://dx.doi.org/10.1109/TCOMM.2009.06.070402 